\title{
SIMULATIONS OF COLLIDING WINDS IN 3 DIMENSIONS
}

\author{
ROLF WALDER \\ Institut für Astronomie, ETH-Zentrum, 8092 Zürich, Switzerland
}

\begin{abstract}
The significance of $3 \mathrm{D}$ effects in simulating colliding winds in WR binaries is demonstrated for the examples V444 Cyg, HD 193793 and $\gamma$ Vel. The effects decrease with increasing separation of the stars. A comparison with symbiotic systems is drawn and a simulation of the symbiotic system EG And is presented.
\end{abstract}

Key words: stars: Wolf-Rayet - hydrodynamics - colliding winds - individual: V444 Cyg - HD 193793 - $\gamma$ Vel - symbiotic systems - individual: EG And

\section{Introduction}

The dynamics of colliding winds are an inherently three-dimensional problem. The topology and geometry of the computed flow pattern show the significance of the orbital motion already in the inner zone of the system. It allows us to gain substantial new insights into the physics of colliding winds and the role they play in the explanation of observed spectra in binary star systems with colliding winds. However, the following remark may be necessary. Simulations in 3D generally suffer from insufficient spatial resolution due to insufficient computer facilities. This may suppress the development of important flow characteristics and may lead to wrong flow patterns. We know for example that instabilities play an important role in many systems where colliding winds are active. But in the 3D simulations performed up to now, the thin shell instability as well as the thermal instability discussed by several authors (Stevens et al. 1992; Dgani et al. 1993; Walder \& Folini, these proceedings) are strongly suppressed compared to $2 \mathrm{D}$ calculations although there is no physical reason to explain their absence.

\section{3D effects: V444 Cyg, $\gamma$ Vel and HD 193793}

V444 Cyg: To illustrate the influence of the orbital motion on colliding winds in WR binaries we performed a simulation of V444 Cyg, which is perhaps the best investigated system of colliding winds with cylindrically symmetric models (Stevens et al. (1992), Shore \& Brown (1988) and Walder (1993)). The orbital period of V444 Cyg is $4.2 \mathrm{~d}$ which leads to an orbital velocity of $290 \mathrm{~km} / \mathrm{s}$ for the WR component and $116 \mathrm{~km} / \mathrm{s}$ for the O-type component. This has to be compared with the terminal velocities of the two winds which were assumed to be $2000 \mathrm{~km} / \mathrm{s}$. For the simulation it was assumed that the WR component loses $1.4 \cdot 10^{-5} \mathrm{M}_{\odot} / \mathrm{y}$ and the O-type component $10^{-6} \mathrm{M}_{\odot} / \mathrm{y}$. In the following, we concentrate on the discussion of the differences between 

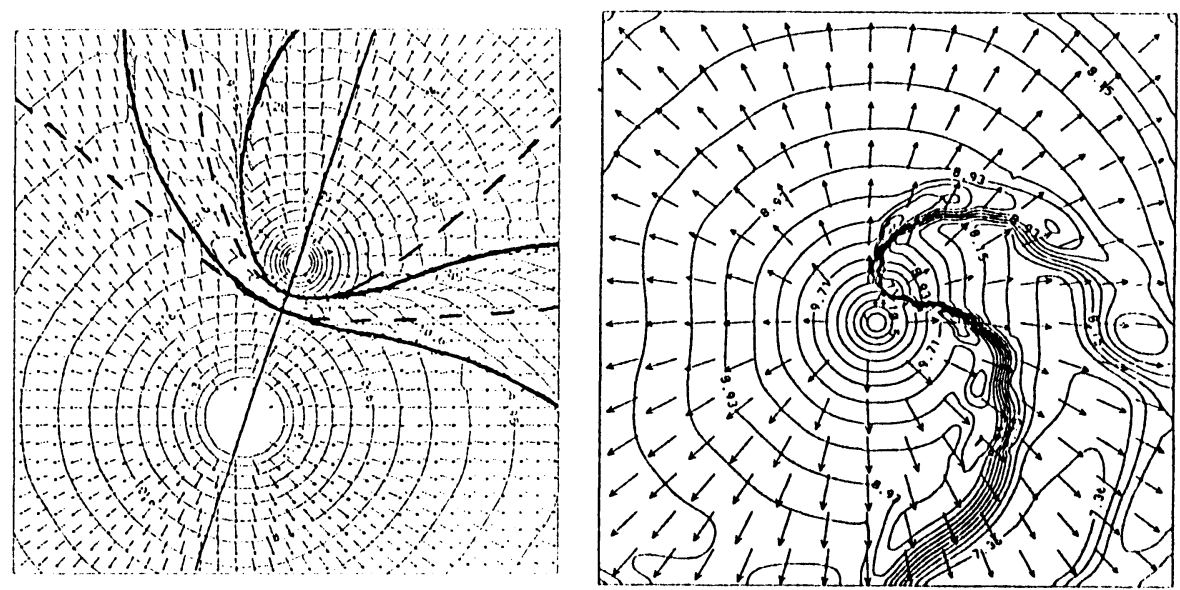

Fig. 1. Colliding winds in V444 Cyg shown in logarithmically scaled density contours and in the velocity field of the orbital plane. Left: The inner part of the interaction zone (bold solid line) resulting from the $3 \mathrm{D}$ adiabatic calculation in comparison with the shape of the zone resulting from an adiabatic cylindrically symmetric simulation. Right: 3D pattern on a larger scale resulting from a simulation in which cooling is self-consistently included. Instabilities are suppressed due to insufficient spatial resolution.

3D and cylindrically symmetric calculations. For a detailed discussion of the other parts of the physics we refer to Stevens et al. (1992).

The influence of the orbital motion to the interaction zone: The most remarkable effect can be seen from the adiabatic simulation (left side of Fig. 1). In 3D the interaction zone has a spiral shape and the two branches of it are both shifted in the direction against the orbital motion. The axis connecting the two stars (which is assumed to be the axis of symmetry in cylindrically symmetric calculations) is cut by the the interaction zone at a distance of approximately one stellar separation from the $\mathrm{O}$-star. The same effect can be seen in a simulation in which radiative cooling was self-consistently included (right side of Fig. 1). Here, the effect is slightly less dramatic because the interaction zone is much more narrow. Furthermore, as we move along the direction normal to the orbital plane, the interaction zone is more and more shifted in the direction opposite to the orbital motion giving the whole zone a significant twist. Within the interaction zone, we do not find much differences in the density and temperature distribution. However, the velocity field is affected by the orbital motion.

One should note that the orbital motion certainly enhances the occurence of turbulences and shocks in the stellar winds themselves. This would also affect the interaction zone because the winds are then less energetic when they met.

$\gamma$ Vel and HD 193793 are intermediate and far separated systems with periods of $78.5 \mathrm{~d}$ and $2900 \mathrm{~d}$, respectively. The calculations show, as can be 
assumed by dimensional arguments, that $3 \mathrm{D}$ effects become less and less important with increasing orbital period (see Fig. 2).
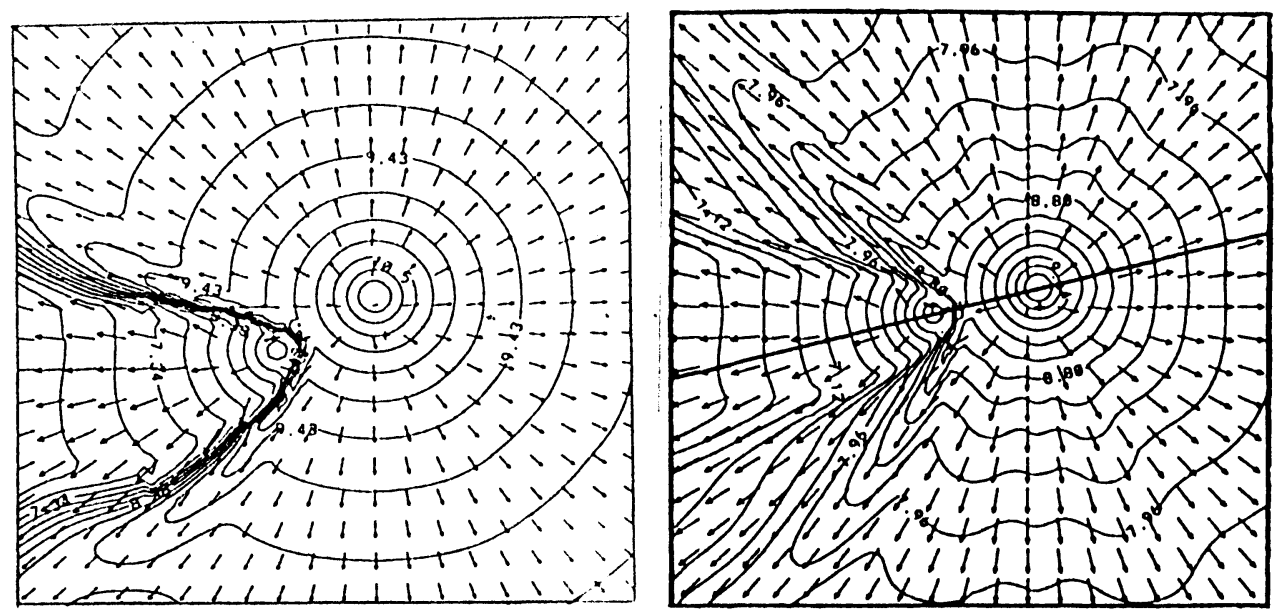

Fig. 2. Interaction zones of colliding winds in the systems $\gamma$ Vel (left) and HD 193793 (right) in periastron. On this scale, in the wide system HD 193793 the orbital motion has only a small effect.

Conclusion: In WR binaries the main effect of the orbital motion on the physics of colliding winds lies in the shape of the interaction zone and not in the production of X-ray radiation. In comparison with cylindrically symmetric calculations, the different relevant column density must affect the calculated spectra. An asymmetry of the X-ray flux in eclipse phases must be present, probably more in the eclipse of the $\mathrm{O}$ component as in the one of the WR component.

\section{A comparison with symbiotic systems}

In the symbiotic system EG And we find conditions typical for narrow symbiotic systems in a state where winds from both stars are present. The relevant system parameters are derived by Vogel (1993): orbital period $P=$ $482 \mathrm{~d}$, eccentricity $e \approx 0$, the primary component is a dwarf-like hot star $\left(T^{*} \approx 75000 \mathrm{~K}\right)$ shedding $5 \times 10^{-9} \mathrm{M}_{\odot} / \mathrm{y}$ by a wind with a terminal velocity of $500 \mathrm{~km} / \mathrm{s}$. The secondary component is a M2.4 III giant with a wind having a terminal velocity of $30 \mathrm{~km} / \mathrm{s}$ and a mass loss rate of $1.5 \times 10^{-8}$ $\mathrm{M}_{\odot} / \mathrm{y}$. With masses of $1.46 \mathrm{M}_{\odot}$ (giant) and of $0.4 \mathrm{M}_{\odot}$ (dwarf) the orbital velocities are $26.2 \mathrm{~km} / \mathrm{s}$ and $7.2 \mathrm{~km} / \mathrm{s}$, respectively.

The main differences with WR systems are: firstly, the wind energies are significantly less powerful than in WR systems; secondly, the characteristics of the two winds in the symbiotic system are highly different; and thirdly, the orbital velocity of the primary is nearly as large as the slow wind velocity. 
As we will see, these differences lead to a completely different flow pattern. In symbiotics, the most important effect of colliding winds is not the $\mathrm{X}$ -

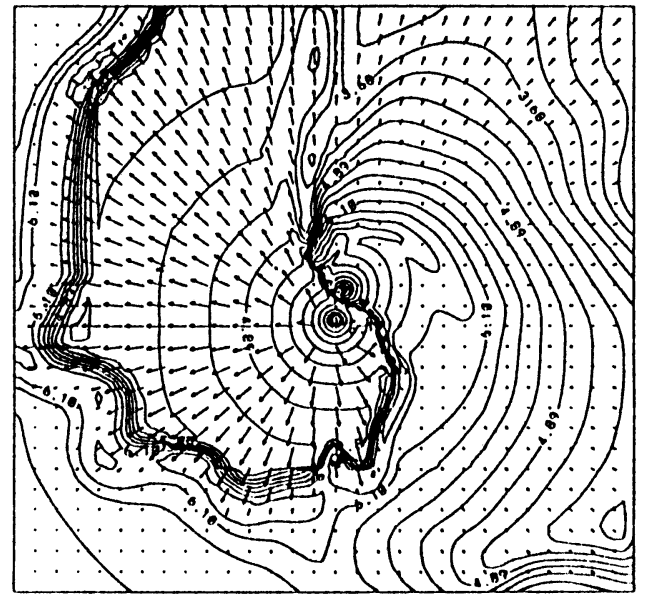

Fig. 3. The symbiotic system EG And shown in density contours and velocity field of the orbital plane. The stars move counter-clockwisely. The pattern is nearly completely determined by the orbital motion.

ray production but the structuralization of the symbiotic nebula due to the fast wind of the dwarf (Walder 1993; Nussbaumer \& Walder 1993). In the symbiotic nebula of EG And shown in Fig. 3, low density (filled with material from the dwarf) and high density zones (filled with material from the giant) can be found. They are separated by the interaction zone of the two winds. This zone is extremely spiral shaped combined with a large twist of the spiral in the direction normal to the orbital plane. The interaction zone is divided into at least three different branches. A spiral-shaped shock is formed where the fast wind pushes the giant wind (left side of Fig. 3). Immediately after the spiraled shock has passed the line connecting the two stars, it is split in two parts. The shocked part of the fast wind can be detected as a jet of moderately hot (some $100000 \mathrm{~K}$ ) material reaching out in space to some separation lenghts (straight upward in Fig. 3). The shocked part of the giant wind is transported outward in an extremely curved spiral, where it is then flowing into the low-density cavity in the wake of the hot star.

\section{References}

Dgani, R., Walder, R., Nussbaumer, H. 1993, $A \& A$ 267, 155

Nussbaumer, H., Walder, R. 1993, A\&A 278, 209

Shore, S.N., Brown, D.N. 1988, ApJ 334, 1021

Stevens, I.R., Blondin, J.M., Pollock, A.M.T. 1992, ApJ 386, 265

Vogel, M. 1993, A\&A (Letters) 274, L21

Walder, R. 1993, PhD thesis, ETH Zürich 


\section{DISCUSSION:}

Owocki: This is very beautiful work and I want to ask about many details. But for now, let me just ask about your adaptive mesh algorithm. Namely, how do you deal with coordinate nonorthogonality in 2-D and 3-D?

Walder: I use Cartesian meshes and the refinement is always done within a rectangle (2D) or a cube (3D). There are some difficulties to correctly compute a spherically symmetric flow on a Cartesian mesh but these are not related to the mesh refinement.

Marchenko: I would like to make a couple of comments which should be important in the modelling of wind-wind collisions.

1. Concerning the variability of the emission or absorption features originating in the bow shock region: the emission peak seems to be much more stable (the variability is barely above the usual instrumental noise of $\sim 1 \%$ ) than the narrow low-shock absorption component. The latter has 3-4 times higher variability.

2. The energetics of the bow shock region in optical region: in Hel lines up to $30 \%$ of the line emission could be generated by shocked gas; at least, $15 \%$ for HeII and up to $100 \%$ for CIII. Koenigsberger: What are the density enhancements you get in the shock cone in V444 Cyg? Walder: In the hot part (not yet cooled) the enhancement is about a factor of 4, in the cooled, compressed part the enhancement is about a factor 30-50.

Shaviv: I assume you must have used the Euler eg. with some artificial viscosity. Please correct me if I am wrong. The question however, is how do you think your results may change if bulk viscosity (say viscosity due to turbulence) is included?

Walder: Yes indeed, I solved the Euler equations with a finite volume method which includes some amount of viscosity implicitely. It is not easy to estimate this amount of viscosity, but it may well be significant. Certainly the transport of mass and angular momentum strongly depend on the viscosity. This may also have consequences for the evolution of the system due to enhanced braking forces. Therefore, the question of viscosity must be carefully studied in future. Cherepashchuk: Did you try to introduce the rotation of WR star in your 3D hydrodynamic calculations? It would be very important for comparison the theoretical orientation of low shock with observations and for empirical determination of axial rotation of WR stars.

Walder: No, not until now. But certainly, stellar rotation is an important aspect and such simulations should be done in the future. 